\title{
BMJ Open Vitamin D supplementation and energy and metabolic homoeostasis in obese and overweight subjects: a protocol for a systematic review
}

\author{
Yu He, ${ }^{1}$ Xiao Yang, ${ }^{2}$ Min Li, ${ }^{2}$ Anren Zhang, ${ }^{2}$ Nianyi Sun (i) ${ }^{1,2}$
}

To cite: He $Y$, Yang $X$,

Li M, et al. Vitamin D

supplementation and energy and metabolic homoeostasis in obese and overweight subjects: a protocol for a systematic review. BMJ Open 2021;11:e051230. doi:10.1136/ bmjopen-2021-051230

- Prepublication history and additional supplemental material for this paper are available online. To view these files, please visit the journal online (http://dx.doi.org/10.1136/ bmjopen-2021-051230)

Received 13 March 2021 Accepted 20 September 2021

A) Check for updates

C Author(s) (or their employer(s)) 2021. Re-use permitted under CC BY-NC. No commercial re-use. See rights and permissions. Published by BMJ.

${ }^{1}$ Department of Rehabilitation, Shengjing Hospital of China Medical University, Shenyang, Liaoning, China

${ }^{2}$ Department of Rehabilitation, Shanghai Fourth People's Hospital, School of Medicine, Tongji University, Shanghai, China

\section{Correspondence to} Dr Nianyi Sun; sny1986@hotmail.com and Professor Anren Zhang; 1518526780@qq.com

\section{ABSTRACT}

Introduction Obesity and vitamin $D$ deficiency are major public health problems. According to the pathophysiological mechanism of obesity as well as the bidirectional relationship between obesity and vitamin D metabolism and storage, vitamin D supplementation in obese and overweight subjects could have beneficial effects on the energy and metabolic homoeostasis. This review will assess the efficacy of vitamin D supplementation on the energy and metabolic homoeostasis in overweight and obese subjects. Methods and analysis In accordance with the Preferred Reporting Items for Systematic Reviews and MetaAnalyses Protocols, we retrieved the relevant literature from the following electronic bibliographic databases: MEDLINE/PubMed, EMBASE and the Cochrane Central Register of Controlled Trials, from inception to June 2021. A manual search of the reference lists of all the relevant research articles will be performed to identify additional studies. We will include randomised controlled trials (RCTs) published in English that examine the effects of vitamin D supplementation on energy and metabolic homoeostasis in overweight and obese subjects. RCTs with multiple vitamin $\mathrm{D}$ groups will also be included. Two reviewers will independently complete the article selection, data extraction and rating. The bias tool from the Cochrane Handbook for Systematic Reviews of Interventions was used to assess the methodological quality of the included studies. A narrative or quantitative synthesis will be performed based on the available data. The planned start and end dates for the study were 1 February 2021 and 1 March 2022.

Ethics and dissemination Ethical approval will not be required for this review. The results of this review will be disseminated in a peer-reviewed journal.

PROSPERO registration number CRD42021228981.

\section{INTRODUCTION}

The definition of overweight and obesity is abnormal or excessive fat accumulation that may impair health. ${ }^{1}$ With the continued increase in the prevalence worldwide, overweight and obesity have been described as a global pandemic. ${ }^{2-5}$ Since 1975 , the worldwide prevalence of obesity has nearly tripled. ${ }^{1}$ In 2016, over one-third of adults worldwide
Strengths and limitations of this study

- The study will be conducted in accordance with the Preferred Reporting Items for Systematic Reviews and Meta-Analyses and recommendations of the Cochrane handbook, which are well-recognised approaches for conducting and reporting of systematic reviews.

- Two reviewers will independently complete the article selection, data extraction and rating, and possible disagreements will be resolved by discussion or consultation with a third author.

- Different protocols for vitamin D supplementation may lead to a large degree of heterogeneity.

- If applicable, prespecified subgroup analyses will be conducted to exclude the differences related to the vitamin D supplementation protocol, comorbid condition or age group.

- When possible, sensitivity analyses will be conducted to test whether the conclusions are robust.

were overweight and $13 \%$ were obese ${ }^{16}$; over 340 million children and adolescents aged above 5 years were overweight or obese. ${ }^{16}$ If this trend continues, it has been projected that up to $57.8 \%$ of the world's adult population could be either overweight or obese by $2030{ }^{7}$ The high prevalence of overweight and obesity, combined with the associated disease burden as well as higher all-cause mortality makes it a global public health challenge. ${ }^{89}$ Moreover, the disease burden of overweight and obesity has been greatly magnified by the current COVID-19 pandemic, as overweight and obesity were represented as an unfavourable factor for COVID-19 severity and mortality. ${ }^{10-12}$

Vitamin D deficiency is another important public health issue, which often coexists with obesity. ${ }^{1314}$ The inverse association between the body mass index (BMI) and vitamin D status (serum 25-hydroxyvitamin D $[25(\mathrm{OH})$ D] concentration) has been suggested irrespective of age, sex, latitude, population group 
or cut-offs to define vitamin D deficiency, ${ }^{13} 1516$ which may be related to a bidirectional relationship between the adipose tissue and vitamin $\mathrm{D}$ metabolism, storage and action. ${ }^{17-23}$ Obesity has been shown to involve a chronic state of low-grade inflammation that dysregulates glucose, lipid and energy metabolism, termed metaflammation. ${ }^{24-26}$ In addition to the metabolic dysregulation in the major peripheral organs that control the energy flux, ${ }^{27}$ metaflammation disturbs the brain function, especially affecting the brain areas that regulate energy and metabolic homoeostasis, such as the hypothalamus. ${ }^{28-32}$ It has been suggested that vitamin $\mathrm{D}$ could play a role in antiobesity, which at least was partly mediated by the vitamin $\mathrm{D}$ receptor in the adipocytes/peripheral organs ${ }^{33-36}$ and the brain. ${ }^{36-38}$ Thus, this has given rise to the hypothesis that vitamin D supplementation in obese and overweight subjects could have beneficial effects on their energy and metabolic homoeostasis.

The assessment of vitamin D supplementation in obese and overweight subjects has been gaining increasing attention in recent randomised clinical trials (RCTs). Several earlier reviews and meta-analyses of RCTs have examined the effect of vitamin D supplementation on weight loss, serum vitamin D concentration and inflammatory or glycaemic markers in overweight and obese individuals with or without comorbid conditions, with limited and less conclusive results. ${ }^{13}{ }^{33}{ }^{39-45}$ However, the energy and metabolic homoeostasis-related biomarkers have not been clearly and fully investigated in the above studies. Therefore, we sought to undertake a comprehensive systematic review of RCTs to evaluate the efficacy of vitamin D supplementation on energy and metabolic homoeostasis in overweight and obese subjects.

\section{METHODS}

\section{Study registration}

This protocol was developed according to the Preferred Reporting Items for Systematic Reviews and Meta-Analyses Protocols (PRISMA-P). ${ }^{46}$ The final study was developed in accordance with the PRISMA statement ${ }^{47}$ under the guidance of the Cochrane Handbook for Systematic Reviews of Interventions. ${ }^{48}$

\section{Inclusion criteria for study selection}

Studies will be included for review if they meet the following inclusion criteria:

\section{Participants}

The adult participants were defined as being overweight or obese (BMI $\geq 25 \mathrm{~kg} / \mathrm{m}^{2}$ (overweight), BMI $\geq 30 \mathrm{~kg} / \mathrm{m}^{2}$ (obese) ). ${ }^{49}$ No restrictions will be assigned with regard to the sex, race, geographical distribution and diseases of the participants enrolled in the study.

\section{Intervention}

Participants in the experimental group were treated with vitamin D supplementation. Any vitamin D and its analogue supplementation will be qualified. There will be no limitations on the routes of administration (oral or intramuscular), dose and duration.

\section{Comparison}

No vitamin D supplementation under the same treatment programme, placebo or sham control.

\section{Outcome measures}

Primary outcomes: The energy metabolism outcomes, such as the total energy expenditure, resting metabolic rate, resting energy expenditure, basal and maximal oxygen consumption rate, bioenergetic health index (BHI), glucose and lipid metabolism outcomes, such as the fasting plasma concentration of glucose and insulin, homoeostasis model assessment for insulin resistance (HOMA), HOMA for $\beta$-cell function, glycated haemoglobin, lipid (cholesterol and triglycerides) profiles and plasma levels of adipokines (adiponectin and leptin). The secondary outcomes included anthropometric and body composition parameters, such as height, weight, waist to hip ratio, BMI, fat mass, fat-free mass, serum $25(\mathrm{OH}) \mathrm{D}$ concentration and adverse events.

\section{Study design and language}

We will include only RCTs published in English.

Studies will be excluded if they were quasi-randomised trials and other types of studies, reported in books, conference proceedings, dissertations or did not have available data for analysis.

\section{Search methods for the identification of studies}

We will retrieve relevant literature across the following electronic bibliographic databases: MEDLINE/PubMed, EMBASE and Cochrane Central Register of Controlled Trials, from inception to June 2021. A search will be conducted using a combination of medical subject heading (MeSH) terms, free-text words and Boolean operators. The concepts of 'participants', 'intervention' and 'RCTs' will be combined with the 'AND' operator. The participants will be defined as overweight and obese subjects, and the intervention is defined as vitamin D supplementation. For each concept, we will combine synonyms and MeSH terms with the 'OR' operator. We will be developing a search strategy for MEDLINE via Ovid (see online supplemental material appendix 1-Search Strategy Example) and adapt this strategy for the other databases. A manual search of the reference lists of all the relevant research articles will be performed to identify additional studies.

\section{Data collection}

Study selection

The bibliographic software Endnote (V.X7) will be used to store, organise and manage all the references. After the removal of duplicate articles, the titles, abstracts and keywords of the retrieved articles will be screened independently by two authors (NS and $\mathrm{YH}$ ) with predefined criteria to identify the eligible studies. After preliminary 


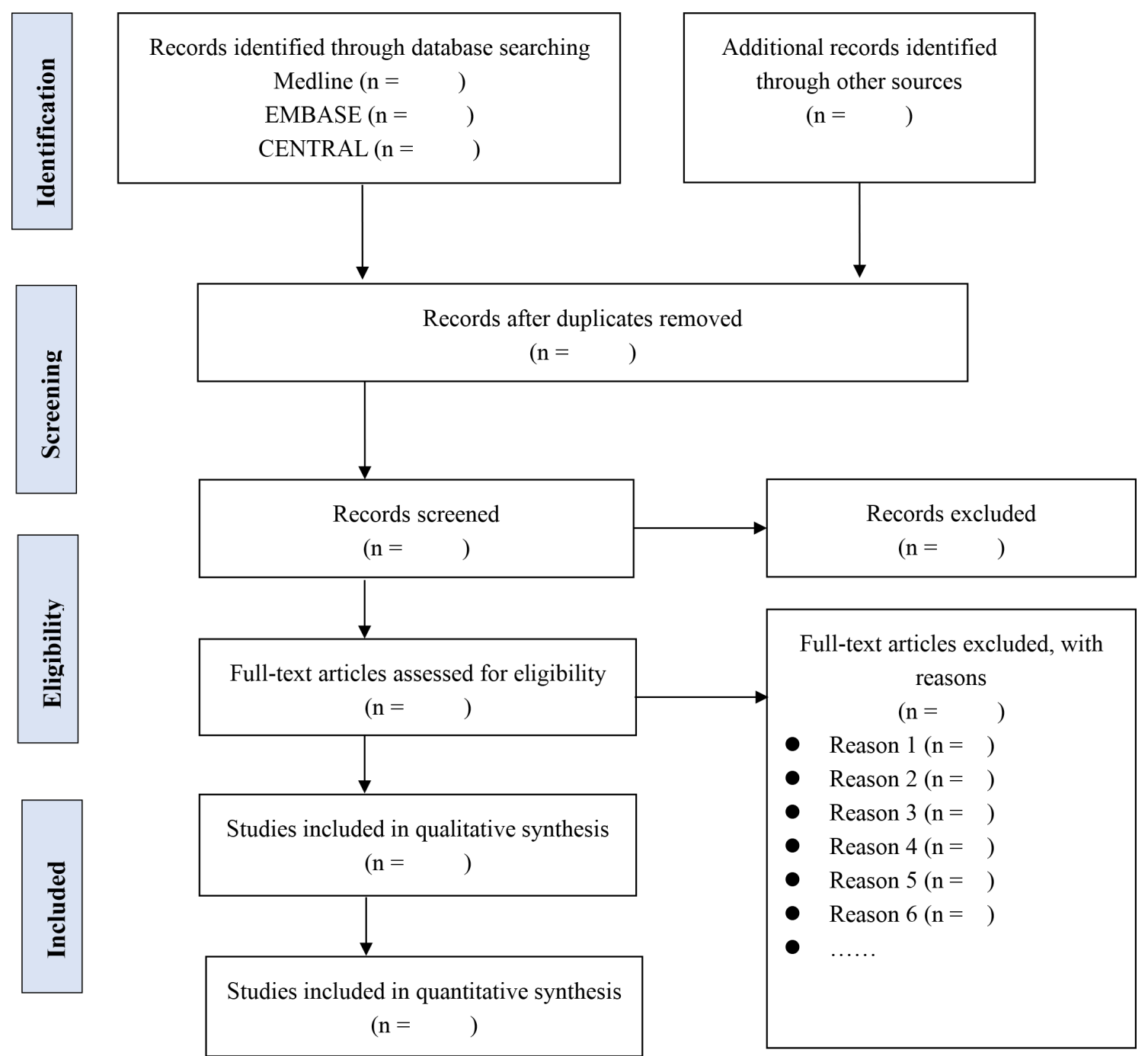

Figure 1 Flow chart of the study selection procedure. CENTRAL, Cochrane Central Register of Controlled Trials.

screening, we will review the full text of potentially eligible articles in detail, to further assess the eligibility, and the reasons for exclusion will be recorded. Any disagreement between the two review authors will be resolved by discussion or consultation with a third author. The final selection procedure will be following the PRISMA guidelines, ${ }^{47}$ and is presented in figure 1.

\section{Data extraction and management}

Two authors (NS and YH) will independently extract the relevant data from the selected studies using a predefined data acquisition form. The extracted data will include the following items:

1. General information: The first author, title, journal, publication year, country, study setting, ethical approv$\mathrm{al}$, trial registration and the funding source.

2. Trial characteristics: study design, method of randomisation, allocation concealment, incomplete outcome data and blinding (participants, researchers and outcome assessors).

3. Intervention: intervention (type, form, dose and duration of vitamin D supplement provided) and compari- son intervention (form, dose, and duration of placebo provided).

4. Participants: Participant demographics, baseline characteristics, inclusion/exclusion criteria, total number and number in each group, and the assessment of compliance and withdrawals.

5. Outcomes and related information: primary and other outcomes, adverse events, duration of follow-up, and intention-to-treat (ITT) analysis.

Possible discrepancies will be resolved through discussion or consultation with a third author. If necessary, we may also contact the original authors for additional relevant information.

\section{Assessment of risk of bias in included studies}

This review will use the bias tool from the Cochrane Handbook for Systematic Reviews of Interventions as the methodological criteria. ${ }^{48}$ The risk of bias for the selected trials will be independently assessed by two authors (NS and $\mathrm{YH}$ ) based on the following criteria: random sequence generation, allocation concealment, blinding of participants, researchers and outcome assessors, incomplete 
outcome data, selective reporting and other sources of bias. The trials will be rated as low risk, unclear risk or high risk, or in each domain after evaluation. Possible disagreements will be resolved through discussion or consultation with a third author.

\section{Data analysis and synthesis}

The Cochrane Review Manager software (V.5.3) will be used for the meta-analysis. In our study, a meta-analysis concerning the effects of vitamin D supplementation will be conducted if two or more studies used the same outcome measure or measured similar constructs.

The summary results are computed in different ways by the data type. Continuous data will be analysed using standardised mean differences with $95 \%$ CI, while the OR with 95\% CI will be computed to analyse the dichotomous data.

Heterogeneity across the studies will be analysed using the $\chi^{2}$ test and $\mathrm{I}^{2}$ statistic. ${ }^{450}$ If $\mathrm{p}>0.1, \mathrm{I}^{2}<50 \%$, a fixed effects model will be used; if $\mathrm{p}>0.1, \mathrm{I}^{2} \geq 50 \%$, a random effects model will be used, substantial heterogeneity is considered in this case; if $p \leq 0.1$, statistical significance is considered, and a subgroup analysis or a narrative description will be carried out. ${ }^{48}$

If applicable, prespecified subgroups will be conducted to explore factors that might impact the strength of the effect, such as the type of vitamin D supplement; form of vitamin D supplement; whether a comorbid condition exists or not and the age group. ${ }^{48}$

When possible, we will perform sensitivity analyses on the following factors to explore the influence of the study quality on the outcomes, such as allocation concealment, blinding of the outcome assessors, drop-out and ITT analysis. ${ }^{48}$

If more than ten trials are included in a result of a metaanalysis, a funnel plot will be constructed to assess the potential publication bias. ${ }^{48}$

The quality of the evidence will be evaluated using GRADEpro software (V.3) at four levels (high, moderate, low or very low) according to the Grading of Recommendations Assessment, Development and Evaluation (GRADE) system. ${ }^{51}$ Two authors (NS and YH) will evaluate the quality of the evidence using GRADE, and possible discrepancies will be resolved through discussion or consultation with a third author.

\section{Patient and public involvement}

This systematic review protocol does not directly involve patients or the general public. The data will be collected from published articles retrieved from the main databases and manual searches.

\section{Ethics and dissemination}

Ethical approval will not be required for the performance of this review protocol. The results of this research will be disseminated in a peer-reviewed journal.

\section{DISCUSSION}

This protocol was registered prospectively in PROSPERO and developed in accordance with the PRISMA-P.
This review systematically and comprehensively assessed the efficacy of vitamin D supplementation on energy and metabolic homoeostasis in overweight and obese subjects. This review protocol provides an overview of the current situation in this area, and we hope that this study will be helpful in providing a valuable reference for future evidence-based and fundamental research to refine vitamin D supplementation in clinical practice and public health.

Acknowledgements We thank the anonymous reviewers for their helpful comments.

Contributors NS, YH and AZ contributed to the conception and design of the study. NS registered the protocol in the PROSPERO database. YH drafted the protocol. NS and $A Z$ revised the protocol critically for important intellectual content. $M L$ and $X Y$ designed the search strategy. NS, YH, AZ, ML and XY participated in the design of data acquisition, analysis and interpretation. All authors have read and approved the final protocol. NS is the guarantor of the protocol and the final review.

Funding The authors have not declared a specific grant for this research from any funding agency in the public, commercial or not-for-profit sectors.

Competing interests None declared.

Patient consent for publication Not applicable.

Provenance and peer review Not commissioned; externally peer reviewed.

Supplemental material This content has been supplied by the author(s). It has not been vetted by BMJ Publishing Group Limited (BMJ) and may not have been peer-reviewed. Any opinions or recommendations discussed are solely those of the author(s) and are not endorsed by BMJ. BMJ disclaims all liability and responsibility arising from any reliance placed on the content. Where the content includes any translated material, BMJ does not warrant the accuracy and reliability of the translations (including but not limited to local regulations, clinical guidelines, terminology, drug names and drug dosages), and is not responsible for any error and/or omissions arising from translation and adaptation or otherwise.

Open access This is an open access article distributed in accordance with the Creative Commons Attribution Non Commercial (CC BY-NC 4.0) license, which permits others to distribute, remix, adapt, build upon this work non-commercially, and license their derivative works on different terms, provided the original work is properly cited, appropriate credit is given, any changes made indicated, and the use is non-commercial. See: http://creativecommons.org/licenses/by-nc/4.0/.

ORCID iD

Nianyi Sun http://orcid.org/0000-0002-3524-4828

\section{REFERENCES}

1 World Health Organization (WHO). Obesity and overweight. Available: https://www.who.int/news-room/fact-sheets/detail/obesity-andoverweight [Accessed 30 Dec 2020].

$2 \mathrm{Ng} \mathrm{M}$, Fleming T, Robinson M, et al. Global, regional, and national prevalence of overweight and obesity in children and adults during 1980-2013: a systematic analysis for the global burden of disease study 2013. The Lancet 2014;384:766-81.

3 Meldrum DR, Morris MA, Gambone JC. Obesity pandemic: causes, consequences, and solutions-but do we have the will? Fertil Steril 2017;107:833-9.

4 Blüher M. Obesity: global epidemiology and pathogenesis. Nat Rev Endocrinol 2019;15:288-98.

5 Swinburn BA, Sacks G, Hall KD, et al. The global obesity pandemic: shaped by global drivers and local environments. Lancet 2011;378:804-14.

6 Abarca-Gómez L, Abdeen ZA, Hamid ZA, et al. Worldwide trends in body-mass index, underweight, overweight, and obesity from 1975 to 2016: a pooled analysis of 2416 population-based measurement studies in 128.9 million children, adolescents, and adults. The Lancet 2017;390:2627-42.

7 Kelly T, Yang W, Chen C-S, et al. Global burden of obesity in 2005 and projections to 2030. Int J Obes 2008;32:1431-7.

8 Abdelaal M, le Roux CW, Docherty NG. Morbidity and mortality associated with obesity. Ann Transl Med 2017;5:161. 
9 Di Angelantonio E, Bhupathiraju S, et al, Global BMI Mortality Collaboration. Body-mass index and all-cause mortality: individualparticipant-data meta-analysis of 239 prospective studies in four continents. Lancet 2016;388:776-86.

10 Földi M, Farkas N, Kiss S, et al. Obesity is a risk factor for developing critical condition in COVID-19 patients: a systematic review and meta-analysis. Obes Rev 2020;21:e13095.

11 Huang Y, Lu Y, Huang Y-M, et al. Obesity in patients with COVID-19: a systematic review and meta-analysis. Metabolism 2020;113:154378.

12 Wadman M. Why obesity worsens COVID-19. Science 2020;369:1280-1.

13 Golzarand M, Hollis BW, Mirmiran P, et al. Vitamin D supplementation and body fat mass: a systematic review and meta-analysis. Eur $J$ Clin Nutr 2018;72:1345-57.

14 Pereira-Santos M, Costa PRF, Assis AMO, et al. Obesity and vitamin D deficiency: a systematic review and meta-analysis. Obes Rev 2015;16:341-9.

15 Rafiq S, Jeppesen P. Body mass index, vitamin D, and type 2 diabetes: a systematic review and meta-analysis. Nutrients 2018;10:1182.

16 Vimaleswaran KS, Berry DJ, Lu C, et al. Causal relationship between obesity and vitamin $\mathrm{D}$ status: bi-directional mendelian randomization analysis of multiple cohorts. PLoS Med 2013;10:e1001383.

17 Hyppönen E, Boucher BJ. Adiposity, vitamin D requirements, and clinical implications for obesity-related metabolic abnormalities. Nutr Rev 2018;76:678-92.

18 Ding C, Gao D, Wilding J, et al. Vitamin D signalling in adipose tissue. Br J Nutr 2012;108:1915-23.

19 Vanlint S. Vitamin D and obesity. Nutrients 2013;5:949-56.

20 Wortsman J, Matsuoka LY, Chen TC, et al. Decreased bioavailability of vitamin D in obesity. Am J Clin Nutr 2000;72:690-3.

21 Song Q, Sergeev IN. Calcium and vitamin D in obesity. Nutr Res Rev 2012;25:130-41.

22 Pourshahidi LK. Vitamin D and obesity: current perspectives and future directions. Proc Nutr Soc 2015;74:115-24.

23 Mallard SR, Howe AS, Houghton LA. Vitamin D status and weight loss: a systematic review and meta-analysis of randomized and nonrandomized controlled weight-loss trials. Am J Clin Nutr 2016;104:1151-9.

24 Gregor MF, Hotamisligil GS. Inflammatory mechanisms in obesity. Annu Rev Immunol 2011;29:415-45.

25 Hotamisligil GS. Inflammation, metaflammation and immunometabolic disorders. Nature 2017;542:177-85.

26 Hotamisligil GS. Inflammation and metabolic disorders. Nature 2006;444:860-7.

27 Muoio DM, Newgard CB. Obesity-related derangements in metabolic regulation. Annu Rev Biochem 2006;75:367-401.

28 Jais A, Brüning JC. Hypothalamic inflammation in obesity and metabolic disease. J Clin Invest 2017;127:24-32.

29 Seong J, Kang JY, Sun JS, et al. Hypothalamic inflammation and obesity: a mechanistic review. Arch Pharm Res 2019;42:383-92.

30 Morton GJ. Hypothalamic leptin regulation of energy homeostasis and glucose metabolism. J Physiol 2007;583:437-43.

31 Pimentel GD, Ganeshan K, Carvalheira JBC. Hypothalamic inflammation and the central nervous system control of energy homeostasis. Mol Cell Endocrinol 2014;397:15-22.

32 Timper K, Brüning JC. Hypothalamic circuits regulating appetite and energy homeostasis: pathways to obesity. Dis Model Mech 2017;10:679-89.
33 Wamberg L, Pedersen SB, Rejnmark L, et al. Causes of vitamin D deficiency and effect of vitamin D supplementation on metabolic complications in obesity: a review. Curr Obes Rep 2015;4:429-40.

34 Wimalawansa SJ. Associations of vitamin D with insulin resistance, obesity, type 2 diabetes, and metabolic syndrome. J Steroid Biochem Mol Biol 2018;175:177-89.

$35 \mathrm{Su} \mathrm{H}$, Lou Y, Fu Y, et al. Involvement of the vitamin D receptor in energy metabolism revealed by profiling of lysine Succinylome of white adipose tissue. Sci Rep 2017;7:14132.

36 Bouillon R, Carmeliet G, Lieben L, et al. Vitamin D and energy homeostasis: of mice and men. Nat Rev Endocrinol 2014;10:79-87.

$37 \mathrm{Xu}$ Y, O'Malley BW, Elmquist JK. Brain nuclear receptors and body weight regulation. J Clin Invest 2017;127:1172-80.

38 Sisley SR, Arble DM, Chambers AP, et al. Hypothalamic vitamin D improves glucose homeostasis and reduces weight. Diabetes 2016;65:2732-41.

39 Chandler PD, Wang L, Zhang X, et al. Effect of vitamin D supplementation alone or with calcium on adiposity measures: a systematic review and meta-analysis of randomized controlled trials. Nutr Rev 2015;73:577-93.

40 Jamka M, Woźniewicz M, Walkowiak J, et al. The effect of vitamin D supplementation on selected inflammatory biomarkers in obese and overweight subjects: a systematic review with meta-analysis. Eur $J$ Nutr 2016;55:2163-76.

41 Jamka M, Woźniewicz M, Jeszka J, et al. The effect of vitamin D supplementation on insulin and glucose metabolism in overweight and obese individuals: systematic review with meta-analysis. Sci Rep 2015;5:16142.

42 Zuk A, Fitzpatrick T, Rosella LC. Effect of vitamin D3 supplementation on inflammatory markers and glycemic measures among overweight or obese adults: a systematic review of randomized controlled trials. PLoS One 2016;11:e0154215.

43 Perna S. Is vitamin D supplementation useful for weight loss programs? A systematic review and meta-analysis of randomized controlled trials. Medicina 2019;55:368.

44 Duan L, Han L, Liu Q, et al. Effects of vitamin D supplementation on general and central obesity: results from 20 randomized controlled trials involving apparently healthy populations. Ann Nutr Metab 2020;76:153-64.

45 de Oliveira LF, de Azevedo LG, da Mota Santana J, et al. Obesity and overweight decreases the effect of vitamin $D$ supplementation in adults: systematic review and meta-analysis of randomized controlled trials. Rev Endocr Metab Disord 2020;21:67-76.

46 Shamseer L, Moher D, Clarke M, et al. Preferred reporting items for systematic review and meta-analysis protocols (PRISMA-P) 2015: elaboration and explanation. BMJ 2015;349:97647.

47 Moher D, Liberati A, Tetzlaff J, et al. Preferred reporting items for systematic reviews and meta-analyses: the PRISMA statement. PLoS Med 2009;6:e1000097.

48 Higgins JPT, Thomas J, Chandler J. Cochrane handbook for systematic reviews of interventions version 6.1. Available: https:// training.cochrane.org/handbook/current [Accessed 02 Jan 2021].

49 Expert panel on the identification, evaluation, and treatment of overweight in adults. clinical guidelines on the identification, evaluation, and treatment of overweight and obesity in adults: executive summary. Am J Clin Nutr 1998;68:899-917.

50 Higgins JPT, Thompson SG, Deeks JJ, et al. Measuring inconsistency in meta-analyses. BMJ 2003;327:557-60.

51 Guyatt GH, Oxman AD, Vist GE, et al. Grade: an emerging consensus on rating quality of evidence and strength of recommendations. $B M J$ 2008;336:924-6. 